\title{
ATRAZINE EFFICIENCY IN AN ANDISOL AS AFFECTED BY CLAYS AND NANOCLAYS IN ETHYLCELLULOSE CONTROLLED RELEASE FORMULATIONS
}

\author{
M. Cea ${ }^{*}$, P. Cartes, G. Palma and M.L. Mora
}

Center of Plant-Soil Interaction and Natural Resources Biotechnology, Scientific and Technological Bioresource Nucleus, Universidad de La Frontera, P.O. Box 54-D, Temuco, Chile. *Corresponding author: mcea@ufro.cl

\begin{abstract}
Atrazine, a herbicide used for the control of broadleaf weeds of different crops, was incorporated in ethylcellulose controlled release formulations (CRFs) by using the solvent evaporation technique. Allophanic clays and nanoclays were incorporated as matrix modifying agents. The formulations were characterized by scanning electron microscopy (SEM) and infrared spectroscopy (FTIR), and their behavior was determined in aqueous phase. Dissipation studies and soil columns experiments with CRFs were also carried out, and compared with commercial formulations (CFs). In addition, a short-term bioassay was performed to evaluate the effect of CRFs and CFs on the emergence and growth of field mustard (Brassica campestris L.) under greenhouse conditions. The matrix modifiers (clays and nanoclays) showed little influence on the particle morphology and atrazine encapsulation efficiency. Furthermore, these matrix modifiers had a slight effect on the atrazine release into water, its dissipation and its behavior in soil compared with the formulation without them. All CRFs increased the atrazine herbicidal activity and reduced their loss by leaching in soil. Although some similarities were found in the efficiency to reduce the seedling emergence between CRFs and CFs, a greater death of seedlings was observed in the CRFs, especially when nanoclays were added into the formulation. These facts denote an advantage of CRFs over CFs due to prolonged bio-efficiency, and longer applications intervals will be produced, minimizing the harmful impact on the environment.
\end{abstract}

Keywords: Atrazine, natural clays and nanoclays, controlled release, soil, herbicidal activity.

\section{INTRODUCTION}

Among herbicides, atrazine, a selective pre- and post-emergence herbicide has been extensively used in forestry, grasslands, maize, sugarcane and noncrop areas. Atrazine and its degradation products constitute potential contaminants (Abdelhafid et al., 2000; Müller et al., 2002), and a frequency of water contamination of about $38 \%$ with variable concentrations from 0.1 to $27 \mathrm{mg} \mathrm{L}^{-1}$ (Costa et al., 2004) could present.

The undesirable side effects arising from the use of conventional agricultural chemicals like atrazine have been minimized by the introduction of polymer supports constituting controlled release 
formulations. The delivery of agrochemicals by controlled release formulations is a promising concept offering advantages for health, nutrition, environmental pollution control, and economic development. In this sense, it has been reported that the microencapsulation of atrazine by different polymers showed excellent controlled release properties (Dailey et al., 1993; Gerst et al., 1998)

Ethylcellulose, a natural, stable, nontoxic and cheap polymer (Moldenhauer and Nairn, 1990) has been commonly used as additive to foods and in various biomedical, pharmaceutical and biotechnological applications because of its low reactivity. Previous reports showed that pesticide-loaded ethylcellulose microspheres had a high herbicidal efficacy (Dailey et al. 1993; Sopeña et al., 2007a).

On the other hand, chemical surface functionality and surface structure of clays and nanoclays play an important role in many technological application fields. Natural and synthetic clays and nanoclays have an attracting interest due to their potential and successful utilization in controlled release systems as modifying agents (Gerstl et al., 1998; Fernández-Pérez et al., 2000, 2004; Dong and Feng, 2005; Hermosín et al., 2006).

Clays commonly used in controlled release systems include monmorillonite, bentonite, and synthetic silice particles. Most of them have been modified to improve their efficiency in the active ingredient retention. For example, acidification has improved the adsorption capacity of bentonite used in alginate controlled release formulation of atrazine (Fernández-Pérez et al., 2004), reducing the release rate of the active component.

The physico-chemical properties of natural clays like allophane, which is the most abundant clay of Andisols of Southern Chile, make them attractive to be used in pesticides controlled release formulations. Allophane has a high specific surface area and variable charge, and it has been demonstrated that allophane-organic matter associations play a key role in controlling soil nutrient availability (Mora and Canales, 1995) and contaminant behavior (Cea et al., 2005, 2007).

Atrazine is widely used for broad leaf weed control in maize production in Andisols of Southern Chile. The high organic matter content of Andisols and agronomical practices like the application of high animal manure dosages to improve crop production affect the efficacy and fate of this herbicide (Briceño et al., 2008; Aguilera et al., 2009). These facts have led farmers to increase the atrazine doses commonly applied to ensure an adequate weed control, with the consequent increase of the environmental risk.

The aims of this work were: (i) to study the atrazine behavior as ethylcellulose controlled release formulations in an Andisol and (ii) to investigate the improvement of atrazine efficiency in the soil-plant system by the addition of allophanic clays and nanoclays to the controlled release formulations.

\section{MATERIAL AND METHODS}

\section{Materials}

Technical atrazine (90\% purity) was kindly provided by Agrícola Nacional SACEI (ANASAC), Chile. Commercial formulations of atrazine (Atranex 90WG and Atrazina $500 \mathrm{SC}$ ) were also tested. Ethylcellulose (EC), $30-50 \mathrm{mPa}$, was purchased from Sigma-Aldrich (St. Louis, USA). Polyvinyl alcohol (PVA, 31-50 
$\mathrm{kDa}, 87 \%$ hydrolysed) was obtained from Sigma-Aldrich (St. Louis, USA). All reagents were of analytical grade. HPLCgrade acetonitrile, methanol and dichloromethane were purchased from J.T. Baker (USA).

Clays and nanoclays were extracted from an Andisol of Temuco Series in Southern Chile. The characterization of clays and nanoclays from this site was previously reported by Calabi et al. (2008).

Soil samples $(0-20 \mathrm{~cm}$ depth $)$ were collected from an Andisol of Freire Series in Southern Chile. The samples were air dried, sieved through $2 \mathrm{~mm}$ and stored in plastic container until their use. The chemical properties of soil were: $15 \%$ of organic matter, $\mathrm{pH} 5.65$ and $20 \mathrm{mg} \mathrm{kg}^{-1}$ of $\mathrm{P}_{\text {olsen. }}$. The soil chemical analyses were performed according the methodology described by Sadzawka et al. (2006).

\section{Microsphere Preparation Characterization}

Microspheres were prepared by the solvent evaporation technique according to the methodologies described by Fernandez-Urzuno et al. (2000) and Dong and Feng (2005). Briefly, ethylcellulose $(1.2 \mathrm{~g})$ was dissolved in $20 \mathrm{~mL}$ of dichloromethane (DCM). Atrazine $(0.2 \mathrm{~g})$ was dissolved in this polymer solution at $40^{\circ} \mathrm{C}$. The herbicide/polymer solution was emulsified in $200 \mathrm{~mL}$ aqueous solution containing $0.15 \% \mathrm{w} / \mathrm{v}$ PVA and various amount of clays $(0,0.04$ and $0.06 \% \mathrm{w} / \mathrm{v})$ and nanoclays $(0,0.0025,0.005$ and $0.01 \% \mathrm{w} / \mathrm{v})$ under stirring (300 rpm). About two drops of 1-octanol were added to the stirred emulsion to reduce foaming. After $8 \mathrm{~h}$ of stirring, the products were filtered, washed with $200 \mathrm{~mL}$ of distilled water and air-dried at $30^{\circ} \mathrm{C}$.

\section{Characterization by scanning electron microscopy and infrared spectroscopy}

The surface morphology of the microspheres was examined by scanning electron microscopy (SEM) (JEOL JSM6380LV). The microsphere size was measured by image analysis. Infrared spectra of $\mathrm{KBr}$ pellets of pure atrazine, pure ethylcellulose, atrazine microspheres and atrazine microspheres containing either clays or nanoclays were obtained using a Brucker Tensor 27 - FTIR spectrometer.

\section{Determination of encapsulation efficiency and herbicide loading}

In order to determine both the efficiency of the microencapsulation process and the atrazine content of microspheres, samples in triplicate were dissolved in methanol (1 $\left.\mathrm{mg} \mathrm{mL}^{-1}\right)$. Later, the samples were filtered with PTFS membrane $(0.45 \mu \mathrm{m}$ pore size; Millipore) and then analyzed by high performance liquid chromatography (HPLC). Briefly, the samples were injected in a Merck Hitachi L-7110 pump, a Rheodyne 7725 injector with $20 \mu \mathrm{L}$ loop and a Merck Hitachi L-7400 UV detector. The detection was set up at 220 $\mathrm{nm}$ and the column was a reverse phase (Lichrosphere RP18, $5 \mu \mathrm{m}$ and $250 \mathrm{~mm}$ ). The mobile phase consisted of acetonitrile: ammonium acetate $0.001 \mathrm{M}$ (40:60 ratio) with a flow rate of $1 \mathrm{~mL}$ $\min ^{-1}$ at $30^{\circ} \mathrm{C}$.

The encapsulation efficiency (EE) was defined as the percentage of herbicide encapsulated by the microspheres in respect to the herbicide used. The herbicide loading (HL) was calculated from the ratio of herbicide encapsulated by microspheres and the final weight of microspheres. 


\section{Sorption studies}

To determine the effect of clays and nanoclays on the encapsulation process, sorption experiments were conducted as follows: $1 \mathrm{~mL}$ of aqueous potassium chloride solutions $(0.01 \mathrm{M})$ containing initial concentrations of atrazine ranging between 2.5 and $20 \mathrm{mg} \mathrm{L}^{-1}$ were prepared. Amounts of $5 \mathrm{mg}$ of clays were mixed with the atrazine solutions, placed in centrifuging tubes and shaken at $25 \pm 1^{\circ} \mathrm{C}$. Preliminary experiments were conducted for various time intervals to determine the sorption equilibrium. The time required to reach the equilibrium was $48 \mathrm{~h}$. After shaking, the suspensions were centrifuged and filtrated (membrane of $0.45 \mu \mathrm{m}$ ). The atrazine concentration in the supernatant was determined by HPLC.

Due to the incapacity to separate the nanoclays from the aqueous phase the sorption experiments were conducted using a dialysis technique described by Seol and Lee (2000). Spectral / Por 6 dialysis tubing with a molecular weight cut off of 1000 Da was washed in distilled water. Freeze-dried nanoclays samples were dissolved in potassium chloride solution $(0.01 \mathrm{M})$ at a concentration of $100 \mathrm{mg}$ nanoclays $\mathrm{L}^{-1}$. Aliquots of $10 \mathrm{~mL}$ were placed in dialysis tubing, and put in $30 \mathrm{~mL}$ test tubes containing $10 \mathrm{~mL}$ of $(0.01 \mathrm{M})$ potassium chloride solution ranging in herbicide concentration from 0 to $20 \mathrm{mg} \mathrm{L}^{-1}$. Tubes were shaken at $25 \pm$ $1^{\circ} \mathrm{C}$. Each experiment included three tubes without nanoclays to verify that the atrazine concentrations inside and outside the bag after $48 \mathrm{~h}$ were equal.

Atrazine concentration inside and outside dialysis tubing was determined measuring the UV absorbance at $220 \mathrm{~nm}$ in a spectrophotometer Spectronic Genesis $^{\mathrm{TM}}$ 2PC, using an atrazine calibration curve. The difference between atrazine concentration inside and outside dialysis tubing was assumed to be the atrazine bound to nanoclays. To evaluate the sorption capacities of clays and nanoclays, the experimental data were fitted to the empirical Freundlich-type isotherm (Eq.[1]):

$$
C_{s}=K_{f} \times C_{e}^{1 / n}
$$

where $C_{s}$ is the atrazine mass sorbed per mass of soil $\left(\mathrm{mg} \mathrm{kg}^{-1}\right) ; C_{e}$ is the concentration of atrazine remaining in solution $\left(\mathrm{mg} \mathrm{L}^{-1}\right)$ after equilibration; $K_{f}$ is the Freundlich distribution coefficient (L $\mathrm{kg}^{-1}$ ); and $1 / \mathrm{n}$ is an exponential empirical parameter that accounts for non-linearity in sorption behavior (Sposito, 1984).

\section{Water release studies}

To compare the release behavior of different controlled release formulations (CRFs), $10 \mathrm{mg}$ of microspheres (three replicates) were immersed in water (200 $\mathrm{mL})$. The systems were put in a shaker at $25^{\circ} \mathrm{C}$ with stirring at $120 \mathrm{rpm}$. At different time intervals, aliquots of $100 \mu \mathrm{L}$ were removed to determine atrazine by HPLC, as described above. The experiment was stopped once, more than $90 \%$ of the active ingredient initially present in the formulations was released. The release kinetics of atrazine from microspheres was evaluated by fitting the data to the Ritger and Peppas (1987) generalized model (Eq. [2]):

$$
M_{t} / M_{z}=k t^{n}
$$

where $M_{t}$ is the amount of herbicide released from the controlled-release microsphere at time $t, M_{z}$ is the total amount of herbicide encapsulated, $k$ is a constant that incorporates characteristics of the macromolecular network system and the active ingredient, and $n$ is the diffusional exponent that indicates the release mechanism. The time needed for $50 \%$ release of atrazine $\left(t_{1 / 2}\right)$ was calculated from these constants. 


\section{Atrazine dissipation in soil}

Atrazine dissipation in soil from the commercial formulations (Atrazina 500 SC (CF1) and Atranex 90WG (CF2)) and CRFs was studied using the Andisol previously characterized (see above). For this purpose, triplicate soil samples (150 g) at $60 \%$ of water holding capacity (WHC) were placed in plastic pots and mixed with CRFs and commercial formulations at application rate of $1.5 \mathrm{mg}$ atrazine $\mathrm{kg}^{-1}$ of soil. After mixing, the samples were incubated in dark for 18 weeks at $20 \pm 1^{\circ} \mathrm{C}$. The soil moisture level was maintained by periodic application of distilled water. Samples (10 g) were taken from each pot at $0,1,2,5,7,12,18$ weeks after treatment and stored at $-20{ }^{\circ} \mathrm{C}$ until their analysis. Atrazine residues were extracted with methanol and analyzed by HPLC. Linear regression equations describing the relationship between atrazine soil concentration $(y=$ Ln (ratio of initial concentration)) and time $(x=$ weeks $)$ were used to calculate dissipation half-lives of the formulations as follow (Eq. [3]):

$$
L_{n} C=L_{n} C_{0}-k t
$$

where $C$ is the soil atrazine concentration after $t$ time, $C_{0}$ is the initial herbicide concentration in soil, and $k$ is the dissipation rate constant. The time required to reach an atrazine dissipation of $50 \%\left(t_{1 / 2}\right)$ was also calculated.

\section{Soil column experiments}

To determine atrazine leaching, duplicate undisturbed soil columns were taken with PVC cylinders of $30 \mathrm{~cm}$ length and $11 \mathrm{~cm}$ internal diameter from the previously characterized Andisol. The bottom of each column was filled with a thin layer of glass wool and covered with a PVC cap provided with a $0.5 \mathrm{~cm}$ diameter hole in the center, where the leachates emerged. All columns were saturated with $500 \mathrm{~mL}$ of distilled water, and allowed to drain for $24 \mathrm{~h}$. Then, $1.36 \mathrm{mg}$ of active ingredient from each formulation (CF1, CF2, CRFs), equivalent to field application of $1.44 \mathrm{~kg}$ atrazine $\mathrm{ha}^{-1}$ were applied to the top of soil columns. Later, the columns were covered with glass wool to avoid damage in soil structure by water. The columns were subjected to watering with distilled water at rate of $50 \mathrm{~mL} \mathrm{~d}^{-1}$ during 30 days. The watering rate was equivalent to the rainfalls registered in Region de $\mathrm{La}$ Araucania of Southern Chile in October 2009. Leachates were collected every day, and atrazine concentration was determined by HPLC after sample concentration.

At the end of the experiment, $10 \mathrm{~g}$ (in triplicate) from each column at different depth $(0-10 \mathrm{~cm}, 10-20 \mathrm{~cm}$ and $20-30 \mathrm{~cm})$ were extracted with $20 \mathrm{~mL}$ of methanol, shaken for $1 \mathrm{~h}$ and finally sonicated for 5 min. The suspensions were centrifuged, concentrated $(1 \mathrm{~mL})$, filtered and analyzed by HPLC in order to determine the residual atrazine amount.

\section{Short time bioassay}

To compare the herbicidal activity of atrazine between some selected CRFs (CRF1, CRF2 and CRF5) and commercial formulations (CF1 and CF2), a short time bioassay was carried out using field mustard (Brassica campestris L.). Andisol samples (150 g) at $60 \%$ WHC were put in plastic pots. After that, five field mustard seeds were sown in each pot, and placed in a growth chamber at 20 $\pm 1^{\circ} \mathrm{C}$ with a photoperiod of $16 \mathrm{~h}$. Field doses of the commercial formulations and its equivalent in active ingredient $(1.44 \mathrm{~kg}$ atrazine $\mathrm{ha}^{-1}$ ) of the CRFs were added to the pots. Three pots were used as replicates for each atrazine formulation, and pots without active ingredient served 
as control. During the growth period, the seedlings were watered daily with distilled water. The emerged seedlings of each pot were quantified 7 days after sowing, and the percentage of emerged seedlings was calculated. At the end of the experiment (15 days) the dead seedlings were quantified, and the aerial and roots length of the surviving seedlings was recorded.

\section{Statistical Analysis}

For the column experiment, data were analysed using analysis of variance (ANOVA), and comparisons between averaged values from different treatments were made by the least significant difference (LSD) test at 0.05 probability significance level.

For the short term bioassay, data were subjected to ANOVA, and significantly different means between treatments were separated with the Tukey's multiple test $(P \leq 0.05)$.

\section{RESULTS AND DISCUSSION}

\section{Microsphere Characterization}

Scanning electron microscopy and infrared spectroscopy

SEM micrographs show the shape and surface morphology of CRFs in presence and absence of clays or nanoclays (Figure 1). In all cases, we observed microparticles with plain, highly irregular and rough surfaces. Nevertheless, it is noteworthy that a high percentage of microparticles showed spherical shapes. We also observed that at increasing clays or nanoclays content, the microparticles formulation lost the spherical form and the roughness and irregularity of the surface increased. The size of CRFs diminished as a consequence of the incorporation of clays or nanoclays into the formulations. However, the microparticle size was not affected by the clays or nanoclays content increment.

The infrared spectra of microparticles were compared with pure atrazine, pure polymer (ethylcellulose), clays and nanoclays spectra (Figure 2 a-c). No differences were observed between the CRFs spectra. We noted the existence of some significant IR bands of atrazine in the spectrum of all CRFs at the same wavenumber: at $3267 \mathrm{~cm}^{-1}$ (stretching $\mathrm{N}$ $\mathrm{H}$ secondary amine), $1622 \mathrm{~cm}^{-1}(\mathrm{C}=\mathrm{N}$ conjugated cyclic system), $1555 \mathrm{~cm}^{-1}$ (N$\mathrm{H}$ bending) and $803 \mathrm{~cm}^{-1}$ (stretching $\mathrm{C}$ $\mathrm{Cl})$. The microparticles IR spectra are a sum of pure atrazine and polymer spectra. In this context, El Bahri and Taverdet (2007) found the prevalence IR band characteristics of the active ingredient in their formulations, which also contained ethylcellulose and other polymers as encapsulating agents.

\section{Herbicide loading and encapsulation efficiency}

HL values of CRFs ranged from 7.3 to $9.9 \%$ (Table 1), and they were very different from the theoretical value $(14.28 \%)$. This difference has been attributed by diverse researchers to PVA, the emulsifying agent, which increases the solubility of herbicide in the continuous phase, influencing negatively the herbicide loading in microparticles (Sopeña et al., 2007a, b; FernandezUrrusuno et al., 2000).

EE values for the different CRFs indicated that in all cases the herbicide 

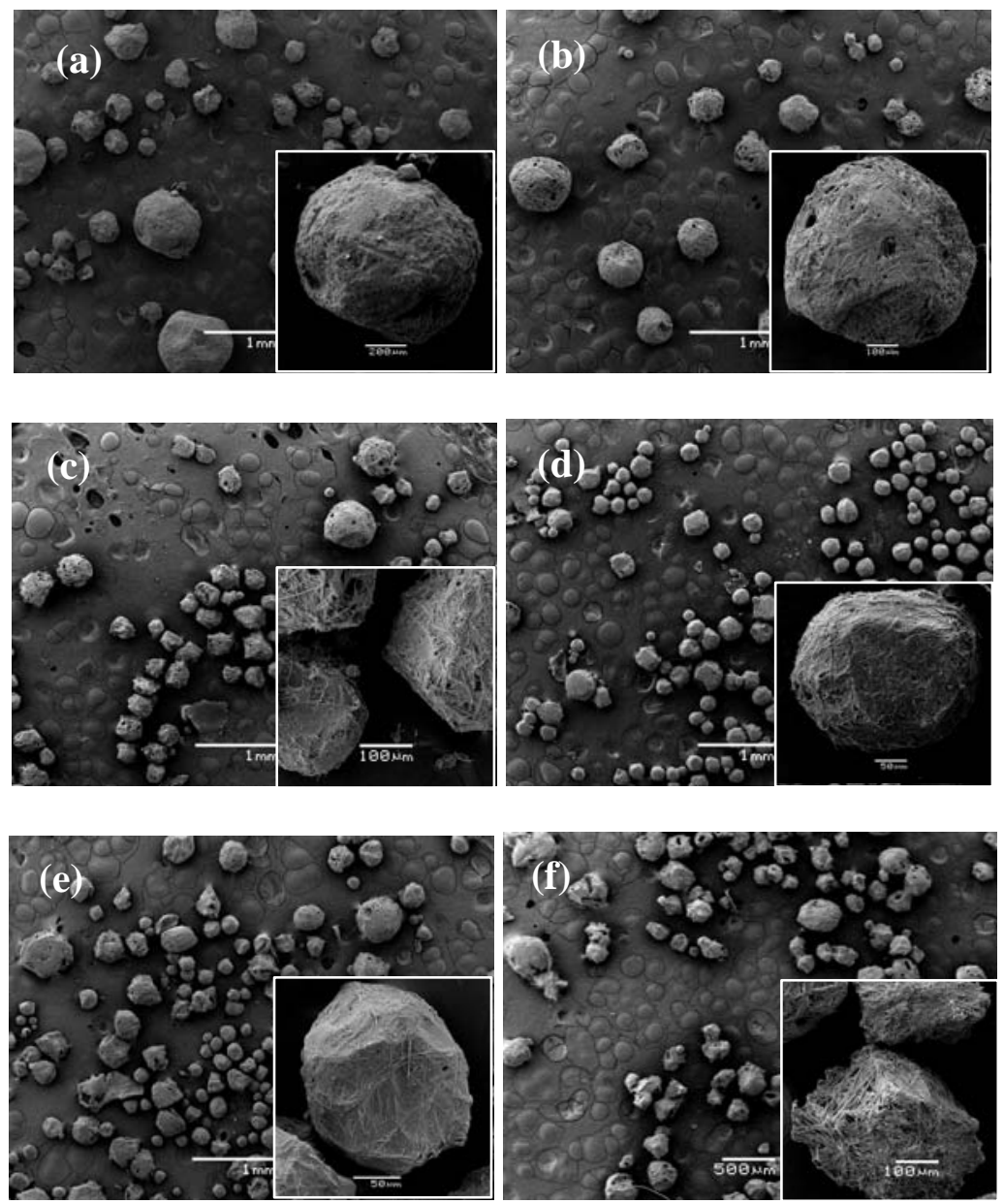

Figure 1. SEM images of the controlled release microparticles of (a) CRF1, (b) CRF2, (c) CRF3, (d) CRF4, (e) CRF5 and (f) CRF6.

content in the microparticles was lower than the theoretical content (Table 1). Although the range of EE values obtained for this parameter is wide $(14-37 \%)$, most of them were around $35 \%$.

The presence of either clays or nanoclays in the aqueous phase seems to have a little influence on $\mathrm{EE}$ at low concentration. At higher than $0.005 \%$ values for nanoclays and $0.04 \%$ for clays, EE value decreased considerably. This phenomenon can be partially explained by the high retention of the active ingredient (atrazine) by clays or nanoclays as it will be discussed later. On the other hand, clays and nanoclays can act as emulsion 

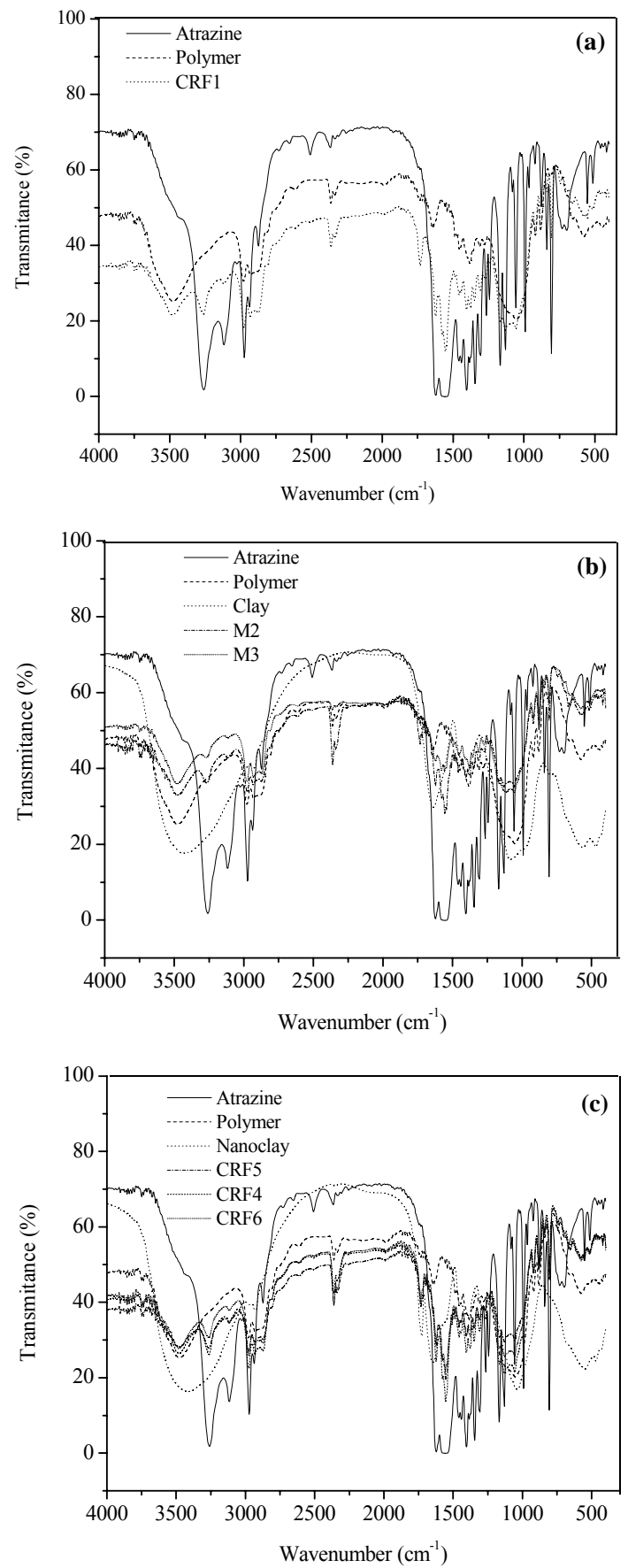

Figure 2. FTIR spectra of controlled release microparticles of (a) CRF1, polymer and atrazine technical grade; (b) CRF2, CRF3, polymer, clays and atrazina technical grade; (c) CRF4, CRF5. CRF6, polymer, nanoclays and atrazine technical grade. 
Table 1. Conditions used during the preparation procedures of formulations, their herbicide loading (HL) and encapsulation efficiency (EE).

\begin{tabular}{lccccc}
\hline Formulation & PVA (\%) & Clay (\%) & Nanoclay (\%) & HL (\%) & EE (\%) \\
\hline CRF1 & 0.15 & 0 & 0 & $8.25 \pm 1.48$ & $35.78 \pm 3.09$ \\
CRF2 & 0.15 & 0.04 & 0 & $9.15 \pm 1.63$ & $37.04 \pm 2.23$ \\
CRF3 & 0.15 & 0.06 & 0 & $8.13 \pm 0.20$ & $13.83 \pm 0.91$ \\
CRF4 & 0.15 & 0 & 0.0025 & $8.05 \pm 0.78$ & $31.48 \pm 2.10$ \\
CRF5 & 0.15 & 0 & 0.005 & $7.30 \pm 0.71$ & $34.53 \pm 4.29$ \\
CRF6 & 0.15 & 0 & 0.01 & $9.90 \pm 0.57$ & $19.26 \pm 2.62$ \\
\hline
\end{tabular}

stabilizers (Dong and Feng, 2005). By analogy, if high concentrations of emulsifiers like PVA can decrease the encapsulation efficiency (FernandezUrrusuno et al., 2000), similar effects of inorganic matrix (e.g. clays or nanoclays) are expected.

\section{Sorption studies}

The sorption isotherms were adequately described by the Freundlich equation (Eq. [1]), with $R^{2}$ values of 0.98 and 0.95 for clays and nanoclays, respectively. The $K_{f}$ values were $2,500 \mathrm{mg} \mathrm{kg}^{-1}$ for clays and $3,981 \mathrm{mg} \mathrm{kg}^{-1}$ for nanoclays indicating the high capacity of both of them to sorb atrazine.

The $1 / n$ values obtained ( 0.73 for clays and 0.78 for nanoclays) showed that atrazine has a strong affinity with the sorbent (Fernández -Perez et al., 2004).

\section{Water release studies}

From the six CRFs, three of them were selected to carry out a water release experiment due to their higher EE. Figure 3 shows the cumulative atrazine release from the selected controlled release formulations (CRF1, CRF2 and CRF5) into water.

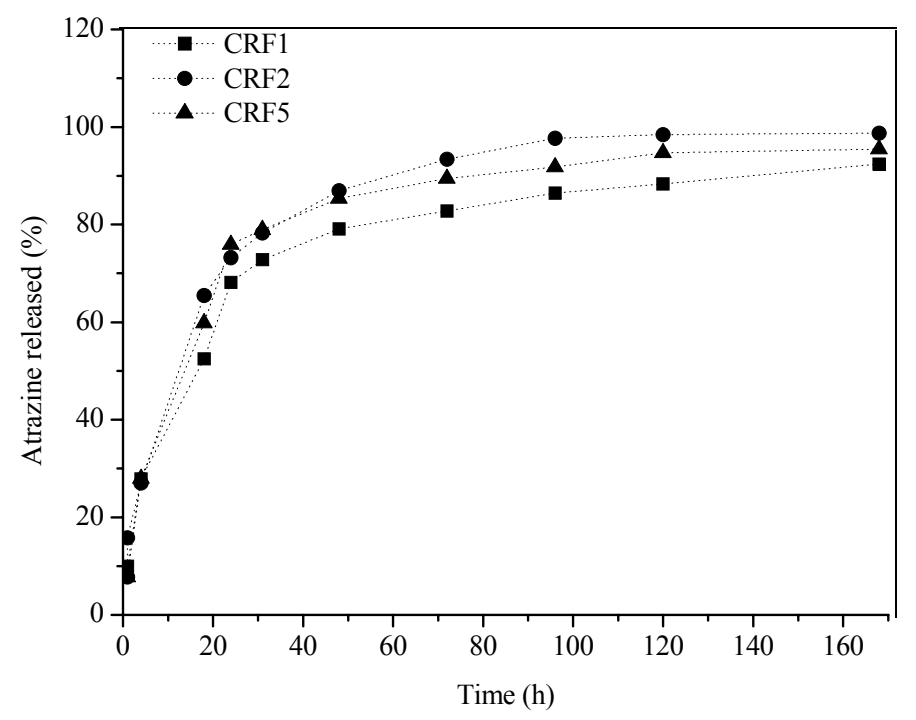

Figure 3. Atrazine released (\%) into water from controlled release formulations. 
It appears that the atrazine release behavior is not markedly affected by the presence of either clays or nanoclays. The values of $k$ and $n$ derived from Equation [2] are shown in Table 2.

Table 2. Constant from fitting the empirical equation $M t / M z=k t^{n}$ to release data of atrazine in water.

\begin{tabular}{lcccc}
\hline Formulation & $k$ & $n$ & $\mathrm{R}^{2}$ & $t_{1 / 2}$ (hours) \\
\hline CRF1 & 0.13 & 0.42 & 0.92 & 24.7 \\
CRF2 & 0.18 & 0.38 & 0.94 & 14.8 \\
CRF5 & 0.12 & 0.47 & 0.88 & 20.8 \\
\hline
\end{tabular}

We observed an increase in the rate $(k)$ of atrazina release in the following order: CRF5 < CRF1 < CRF2. The $n$ values ranged from 0.38 (CRF2) to 0.47 (CRF5). Values of $n$ close to 0.43 are indicatives of a Fickian diffusion mechanism in spherical monolithic matrixes (Ritger and Peppas, 1987). According to FloresCespedes et al. (2007) the differences of $n$ values are a consequence of: (i) the higher complexity of systems containing matrix modifier (e.g. clays), and (ii) the capacity of these agents to interact with the active ingredient. Thus, higher sorption capacity of modifying agent could result in a slower release of active ingredient.

In fact, in our work, the CRF containing nanoclays (CRF5) showed a slight deviation from $n=0.43$, and therefore a slower release of atrazine which is supported by our sorption experiments. In contrast, the presence of clays in the formulation (CRF2) slightly increased the rate of active ingredient release. This result is in agreement with SEM images presented in Figure 1, since in CRF2 the roughness and irregularity of surface was higher compared with those of CRF1 and CRF5. In addition, CRF2 exhibited a greater formation of cracks and pores at the surface than the other ones. Such differences in the surface properties could explain the higher rate of atrazine release in $\mathrm{CRF} 2$ in respect to CRF1 and CRF5.

\section{Dissipation experiment in soil}

In this experiment we used the three before selected CRFs and two commercial formulations (CF1 and CF2). The kinetic studies of atrazine dissipation in soil from different formulations are shown in Figure 4. We observed that in soil treated with $\mathrm{CF} 1$ and $\mathrm{CF} 2$, more than $75 \%$ of the initially applied atrazine was dissipated in 1 week. In contrast, the equivalent level of dissipation for CRFs was reached at 12 weeks, independent from the presence of clays or nanoclays. In general, CRFs protected against herbicide dissipation better than CFs.

Atrazine soil degradation from the different formulations fitted closely to the first-order kinetics evaluated here $(\mathrm{Eq}$ [3]), with regression coefficients ranging from 0.92 to 0.98 (Table 3). Half-life values $\left(t_{1 / 2}\right)$ are also reported in Table 3 . $t_{1 / 2}$ were 1.29 and 1.26 for CF1 and CF2, respectively. These values are comparable with those obtained by Aguilera et al. (2009) in an Andisol with similar chemical characteristics and with previous history of atrazine application, like our specific soil. Comparatively, the $t_{1 / 2}$ values for CRFs were at least 2.79-fold higher than commercial formulations (CF1 and CF2). CRF2 formulation showed a singular behavior. As it can be observed in Table 3, atrazine from this formulation suffered more dissipation than the other CRFs. This behavior could be attributed to the microparticle composition (prepared in presence of clays) and surface irregularity as it was shown in Figure 1. In this way, the presence of pores and cracks in the microparticles surface could make them ore susceptible to environmental damagethan CRF1 and CRF5. 


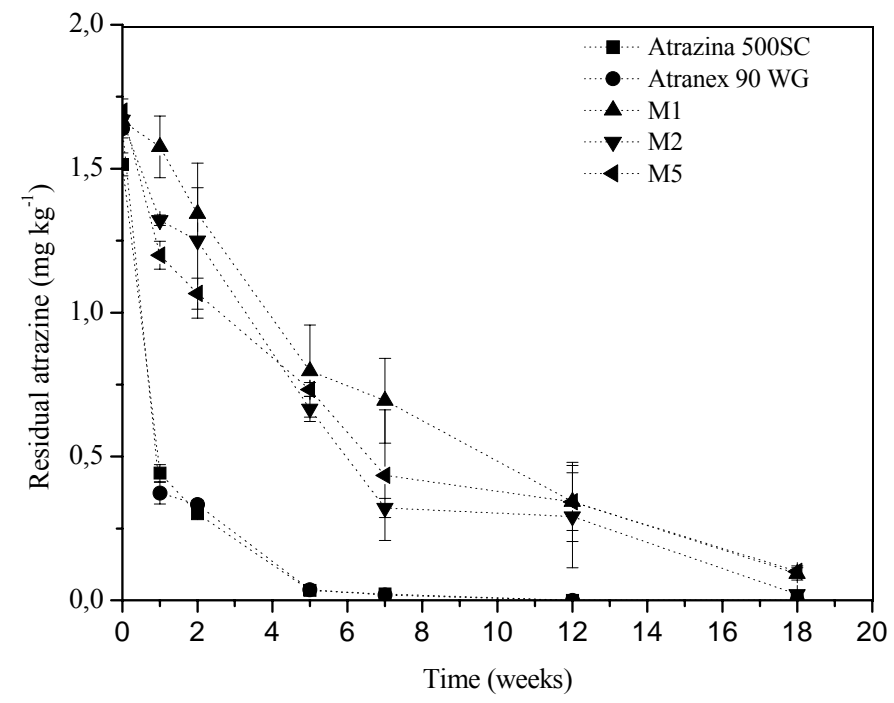

Figure 4. Atrazine dissipation in soil from ethylcellulose microparticles (CRF1, CRF2 and CRF5) and commercial formulations (CF1 and CF2)

Table 3. Half-Life values ( $t_{1 / 2}$ in weeks) in soil dissipation experiment.

\begin{tabular}{lccc}
\hline Formulation & $k$ & $\mathrm{R}^{2}$ & $t_{1 / 2}$ (weeks) \\
\hline CF1 & 0.54 & 0.97 & 1.29 \\
CF2 & 0.55 & 0.97 & 1.26 \\
CRF1 & 0.14 & 0.98 & 4.87 \\
CRF2 & 0.19 & 0.97 & 3.61 \\
CRF5 & 0.16 & 0.97 & 4.26 \\
\hline
\end{tabular}

From our results, it can be inferred that CRFs represent an advantage in comparison with CFs. The lower dissipation given by the encapsulation matrix could result in a greater stabilization of the active ingredient against environmental degradation. Furthermore, according to Tsuji (2001) the active ingredient stabilization produces longer application intervals which results in labor saving.

\section{Soil columns experiment}

The behavior of CFs and CRFs in undisturbed soil was determined by measuring the atrazine content in leachates and soil in a column experiment. At the end of the experiment (30 d), atrazine was not detected in the leachates when the CRFs were applied, and only a small amount $(0.01 \%$ for CF1 and $0.04 \%$ for CF2) of active ingredients, referred to the total herbicide applied, was found by effect of CFs application. The quantity of retained atrazine at the different soil depths is presented in Figure 5. At $0-10 \mathrm{~cm}$ depth, the retained atrazine was at least 3.8-fold higher in CRFs compared with CFs. We did not observe significant differences in the column distribution pattern of atrazine between 
CFs and CRFs, and most of the retained active ingredient was accumulated in the first $10 \mathrm{~cm}$ depth. Previous reports have also shown the greatest retention of pesticides in the upper soil layer (Fernández-Pérez et al., 2001; Sopeña et al., 2007a, b). The total atrazine recovery (soil plus leachates) at $30 \mathrm{~d}$ was no more than $3.25 \%$ for $\mathrm{CFs}$ and $15.49 \%$ for CRFs. The difference to $100 \%$ of the total atrazine applied was attributed to degradation processes.

As annual grasses and small seeded broadleaf weeds germinate in the upper $10 \mathrm{~cm}$ of soil (Sopeña et at., 2007a) our CRFs represent an excellent alternative to CFs, since after $30 \mathrm{~d}$ of application they retained significantly greater amount of active ingredient $(9.81 \%$ of herbicide applied) in the soil surface $(0-10 \mathrm{~cm})$ in comparison with CFs $(1.60 \%)$.

\section{Short-term bioassay}

In the short-term bioassay, the emergence percentage of field mustard was evaluated 7 days after atrazine application as shown in Table 4. All formulations (CFs and CRFs) inhibited the seedling emergence as compared with the control treatment. In average, the CRFs caused greater inhibition of emergence than CFs. Likewise, after 15 days of atrazine addition, the percentage of dead seedlings was at least 3.54 fold higher for the CRFs compared with $\mathrm{CF} 2$, and the highest lethal effect (67\%) was observed when CRF5 was applied. These facts demonstrated that although $\mathrm{CFs}$ and $\mathrm{CRFs}$ caused an immediate herbicide effect after the atrazine application, in CRFs this effect was maintained within the time. In fact, CRFs caused a further later damage in emerged seedlings. In terms of seedling growth, no differences were observed in the aerial length between CFs and CRFs, but CRFs significantly decreased the root length (Figure 6). Thus, the later negative effect of CRFs on root growth increased the lethal effect of atrazine in respect to CFs.

The overall result of atrazine application was an increase of the lethal effect of CRFs over CFs ranging from $13 \%$ to $64 \%$ (Table 4). This is in agreement with the results of dissipation (Figure 4) and column (Figure 5) experiments.

The aerial and root length of seedlings grown in pots treated with CRFs containing either clays (CRF2) or nanoclays (CRF5) were not statistical different from those of CRF1 (ethylcelluloce atrazine formulation). This fact was as it was expected, as similar behavior of CRFs was found in soil columns and dissipation experiments, irrespective of the presence of clays or nanoclays.

Other reports have shown a low herbicidal activity of controlled release formulations on soybean (Sopeña et al., 2007a) and oat (Sopeña et al., 2007b) in the short-term due to the slow discharge of the active ingredient to the soil. Nevertheless, Akelah et al. (2008) found an effective herbicidal activity from their atrazine controlled release formulations over herbs in maize crop, which agrees with the results of our experiment.

Thus, the use of CRFs could provide an advantage over CFs due to a more prolonged bio-efficiency seems to occur as a consequence of the slow release of active ingredient to the soil.

\section{CONCLUSIONS}

Matrix modifiers, such as clays and nanoclays added to controlled release atrazine formulations (CRFs), influenced slightly the particle morphology, 
Atrazine efficiency in an Andisol as affected by clays and nanoclays, Cea et al.

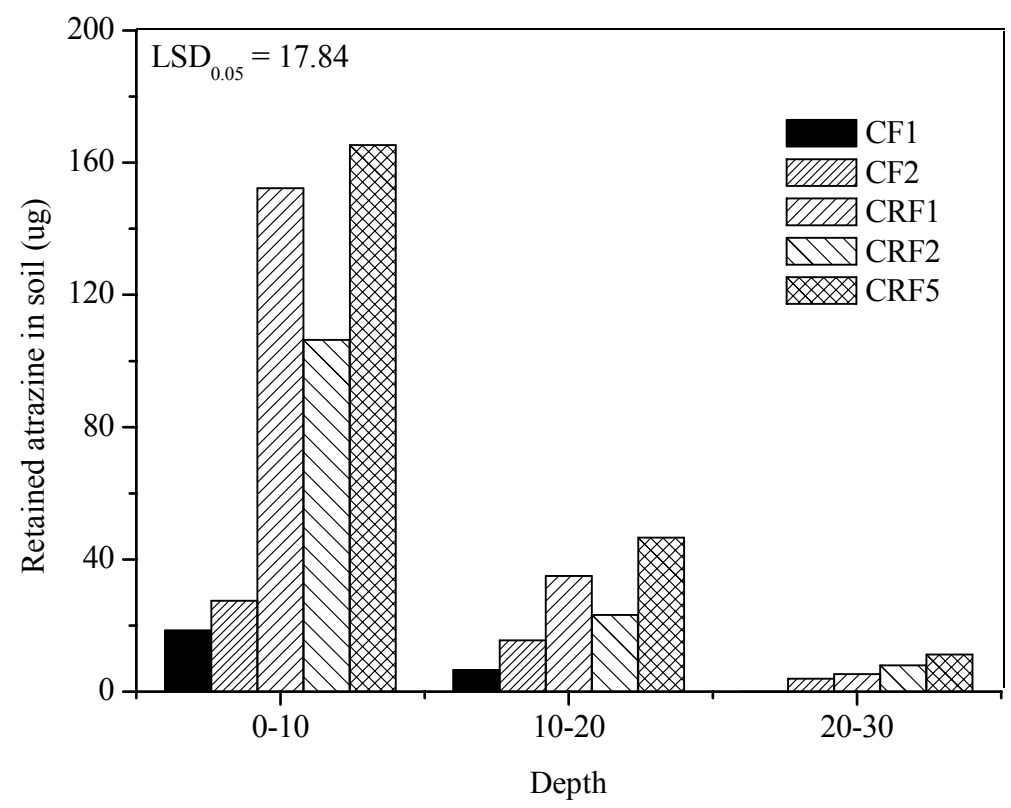

Figure 5. Distribution (micrograms) of atrazine at different depths in the soil columns after leaching the five tested formulations.

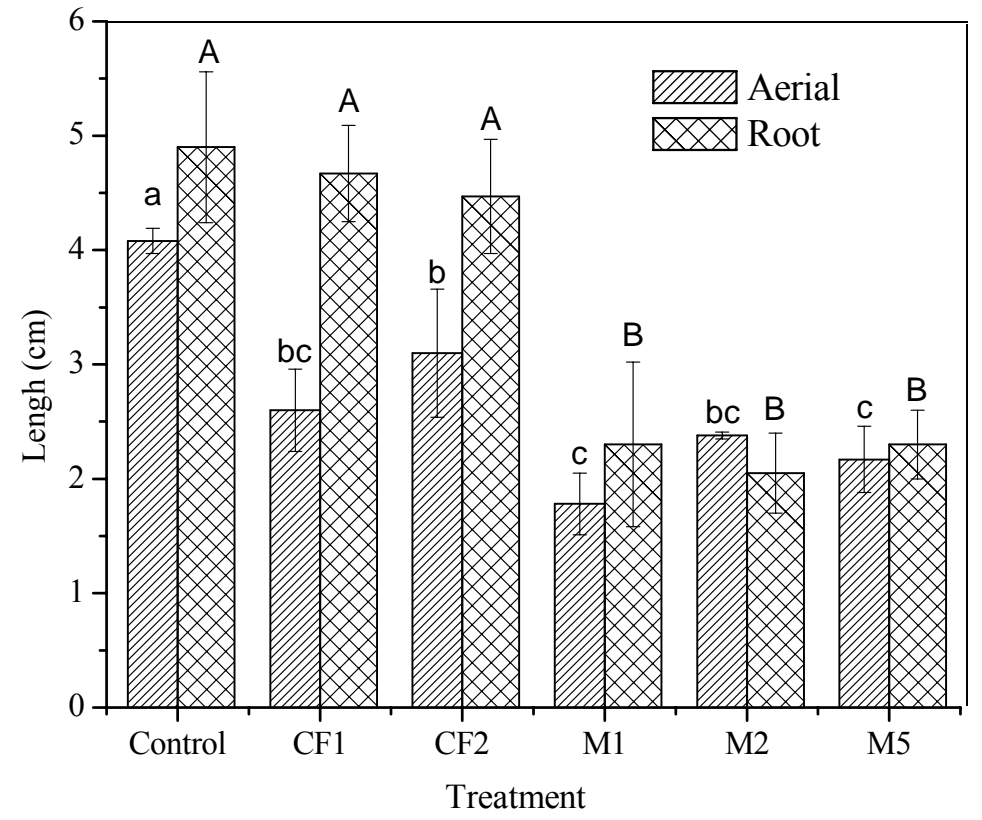

Figure 6. Effect of atrazine formulations on aerial and root length of surviving seedlings after 15 days of application. 
Table 4. The lethal effect on Brassica campestris and its percentage emergence after applying the different formulations

\begin{tabular}{lcc}
\hline Formulation & Dead seedlings (\%) & Emergence of seed (\%) \\
\hline Control & 0 & 67 \\
CF1 & 0 & 33 \\
CF2 & 11 & 53 \\
CRF1 & 44 & 27 \\
CRF2 & 39 & 40 \\
CRF5 & 67 & 40 \\
\hline
\end{tabular}

encapsulation efficiency, the release into water, dissipation and atrazine behavior in soil. Furthermore, no difference in the CRFs herbicidal activity was found as a consequence to the addition of clays or nanoclays into the ethylcellulose formulations.

In all CRFs the atrazine herbicidal activity in soil was increased, and the loss by leaching through the soil profile was reduced. Even though similarities were observed in the efficiency of CRFs and CFs to diminish the seedling emergence, a higher death of seedlings was observed in the CRFs, especially when nanoclays were added into the formulation. These facts indicate an advantage of CRFs over CFs due to prolonged bio-efficiency and longer applications intervals will be produced, minimizing the harmful impact on the environment.

ACKNOWLEDGMENTS. We wish to thank FONDECYT No 3080039 for its financial support.

\section{REFERENCES}

Abdelhafid, R., Houot, S., Barriuso, E. 2000. Dependence of atrazine degradation on $\mathrm{C}$ and $\mathrm{N}$ availability in adapted and nonadapted soils. Soil Biology and Biochemistry $32,389-401$.

Aguilera, P., Briceño, G., Candia, M., Mora, M.L., Demanet, R., Palma, G. 2009. Effect of dairy manure rate and the stabilization time of amended soils on atrazine degradation. Chemosphere 77, 785-790.

Akelh, A., Rehab, A., El-Gamal, M. 2008. Preparation and applications of controlled release systems based on intercalate atrazine salt and polymeric atrazine salt onto montmorillonite clay. Materials Science and Engineering C 28, 1123-1131.

Briceño, G., Demanet, R., Mora, M.L., Palma, G. 2008. Effect of cow manure on Andisol properties and atrazine adsorption. Journal of Environmental Quality 37, 15191526.

Calabi, M., Cea, M., Reyes, P., Jara, A., Sanhueza, S., Mora, M.L. 2008. Comparative study of structural and physical-chemical characteristics of nanoparticles extracted from two Andisols of southern Chile: potential in agricultural application. Journal of Soil Science and Plant Nutrition. 8, 172-173. Special Issue.

Cea, M., Seaman, J.C., Jara, A., Fuentes, B., Mora, M.L., Diez, M.C. 2007. Adsorption behavior of 2,4-dichlorophenol and pentachlorophenol in an Allophanic soil. Chemosphere 67, 1354 - 1360 . 
Cea, M., Seaman, J.C., Jara, A., Mora, M.L., Diez, M.C. 2005. Describing chlorophenol sorption on variable-charge soil using the triple-layer model. Journal of Colloid Interface Science 292, 171-178.

Costa, J., Bedmar, F., Aparicio, V., Daniel, P. 2004. Pérdidas de atrazina desde un monocultivo de maíz en el sudeste bonaerense. In: INTA Agropecuaria (Editor), Buenos Aires.

Dailey, O., Dowler, C., Mullinix, B. 1993. Polymeric microcapsules of herbicides atrazine and metribuzin: preparation and evaluation of controlled release properties. Journal of Agricultural Food Chemistry 41, $1517-1522$.

Dong, Y., Feng, Si-Shen. 2005. Poly(D,Llactide-co-glycolide)/montmorillonite nanoparticles for oral delivery of anticancer drugs. Biomaterials 26, 6068-6076.

El Bahri, Z., Taverdet, J.L. 2007. Elaboration and characterisation of microparticles by pesticide model. Powder Technology 172, 30-40

Fernández-Pérez, M., Flores-Céspedes, F., González-Pradas, E., Villafranca-Sánchez, M., Pérez -García, S., Garrido-Herrera, F. 2004. Use of activated bentonites in controlled-release formulations of atrazine. Journal of Agricultural and Food Chemistry. 52, 3888-3893.

Fernández-Pérez, M., González-Pradas, E., Villafranca-Sánchez, M., Flores-Céspedes, F. 2001. Mobility of atrazine from alginatebentonite controlled release formulations in layered soils. Journal of Agricultural and Food Chemistry 43, 347-353.

Fernández-Pérez, M., González-Pradas, E., Villafranca-Sánchez, M., Flores-Céspedes, F. 2000. Mobility of isoproturon from an alginate-bentonite controlled release formulation in layered soil. Chemosphere 41, 1495-1501.

Fernandez-Urrusuño, R., Gines, J.M., Morillo, E. 2000. Development of controlled release formulations of alachlor in ethylcellulose. Journal of Microencapsulation 17(3), 331-342.
Flores-Céspedes, F., Villafranca-Sánchez, M., Pérez-García, S., Fernández-Pérez, M. 2007. Modifying sorbents in controlled release formulations to prevent herbicides pollution. Chemosphere 69, 785-794.

Gerstl, Z., Nasser, A., Mingelgrin, U. 1998. Controlled release of psticidesinto soils from clay-polymer formulations. Journal of Agricultural and Food Chemistry 46, 37973802 .

Hermosín, M.C., Celis, R., Facenda, G., Carrizosa, M.J., Ortega-Calvo, J.J., Cornejo, J. 2006. Bioavailability of herbicide 2,4-D formulated with organoclays. Soil Biology and Biochemistry 38, 2117-2124.

Moldenhauer, M., Nairn, J.G. 1990. Formulation parameters affecting the preparation and properties of microencapsulated ion-exchanged resins containing theophylline. Journal of pharmaceuticalScience 79,659-666.

Mora, M.L., Canales, J. 1995. Interactions of humic substances with allophanic compounds. Communication in Soil Science and Plant Analysis 26, 2805- 2817.

Müller, K., Bach, M., Hartmann, H., Spiteller, M., Frede, H. 2002. Point- and Nonpoint-Source Pesticide Contamination in the Zwester Ohm Catchment, Germany. Journal of Environmental. Quality 31, 309. 318.

Ritger, P., Peppas, N. 1987. A simple equation fro description of solute release. I. Fickian release from non-swellable devices in the form of slabs, spheres, cylinders and discs. Journal of Controlled Release 5, 23-26.

Sadzawka, A.M. Carrasco, R. Grez, M.L. Mora, H. Flores and A. Neaman. 2006. Métodos de análisis de suelo recomendados para los suelos de Chile. Serie Actas Instituto de Investigaciones Agropecuarias, Ministerio de Agricultura, Santiago-Chile, №34, 164 p.

Seol, Y., Lee, L. 2000. Effect of dissolved organic matter in treated effluents on sorption of atrazine and prometryn by soils. Soil Science Society American Journal 64, 19761983. 
Sopeña, F., Cabrera, A., Maqueda, C.,Morillo, E. 2007a. Ethylcellulose formulations for controlled release of the herbicide alachlor in a sandy soil. Journal of Agricultural and Food Chememistry 55(20), 8200-8205.

Sopeña, F., Maqueda, C., Morillo, E. 2007b.

Norflurazon mobility, dissipation, activity, and persistence in a sandy soil as influenced by formulation. Journal of Agricultural and Food Chemistry 55 (9), 3561-3567.
Sposito, G. 1984. The surface chemistry of soils. Oxford Univ. Press, New York.

Tsuji, K. 2001. Review: Microencapsulation of pesticides and their improved handling safety. Journal of Microencapsulation 18(2), 137-147. 